\title{
Head and neck reconstruction using free flaps: a 30-year medical record review
}

\author{
Joong Min Suh, \\ Chul Hoon Chung, \\ Yong Joon Chang \\ Department of Plastic and \\ Reconstructive Surgery, Kangdong \\ Sacred Heart Hospital, Seoul, Korea
}

\begin{abstract}
Background: The free flap surgical method is useful for the reconstruction of head and neck defects. This study retrospectively analyzed the results of head and neck reconstructions using various types of free flaps over the past 30 years.

Methods: Between 1989 and 2018, a total of 866 free flap procedures were performed on 859 patients with head and neck defects, including 7 double free flaps. The causes of vascular crisis and salvage rate were analyzed, and the total flap survival rate calculated among these patients. Additionally, the survival and complication rates for each flap type were compared.

Results: The 866 cases included 557 radial forearm flaps, 200 anterolateral thigh flaps, 39 fibular osteocutaneous flaps, and 70 of various other flaps. The incidence of the vascular crisis was $5.1 \%$; its most common cause was venous thrombosis (52.3\%). Salvage surgery was successful in $52.3 \%$ of patients, and the total flap survival rate was $97.6 \%$. The success rate of the radial forearm flap was higher than of the anterolateral flap $(p<0.01)$, and the primary sites of malignancy were the tongue, tonsils, and hypopharynx, respectively.

Conclusion: The free flap technique is the most reliable method for head and neck reconstruction; however, the radial forearm free flap showed the highest success rate (98.9\%). In patients with malignancy, flap failure was more common in the anterolateral thigh $(5.5 \%)$ and fibular (5.1\%) flaps.
\end{abstract}

Keywords: Free flaps / Head / Microsurgery / Neck / Reconstructive surgery

\section{INTRODUCTION}

Tissue defects in the head and neck are among the most difficult areas a plastic surgeon should reconstruct. They may occur for a variety of reasons such as extensive resection to treat malignant and benign tumors, trauma, and congenital defects. The head and neck are densely populated with important organs that perform various important functions such as breathing,

Correspondence: Chul Hoon Chung

Department of Plastic and Reconstructive Surgery, Kangdong Sacred Heart

Hospital, 150 Seongan-ro, Gangdong-gu, Seoul 05355, Korea

E-mail: c21ps@hanmail.net

This article was presented at the 10th Research \& Reconstruction Forum on July 24, 2020, in Busan, Korea.

Received December 24, 2020 / Revised February 8, 2021 / Accepted February 9, 2021 mastication, swallowing, and vocalization; therefore, when reconstruction is required, cosmetic and functional results should be carefully considered. Reconstruction using free flaps has increased in popularity following the introduction of free groin flaps by Daniel and Taylor in 1973. Since then, various free flaps were introduced by the development of microsurgical techniques, instruments, and vascular anatomy [1]. Until recently, the success rate of free flaps reported in various studies ranges from $94 \%$ to $96 \%$. And head and neck reconstruction using free flaps has been accepted as the gold standard [2-6]. Therefore, the authors intend to retrospectively analyze and report data on free flaps performed for head and neck reconstruction over the past 30 years. 


\section{METHODS}

This study was approved by the appropriate institutional review board and performed in accordance with the principles of the Declaration of Helsinki. Informed consent was waived because of the retrospective nature of the study and the analysis used anonymous clinical data.

In the approximately 30 years between 1989 and 2018, 859 patients underwent microsurgical reconstruction for their defect in head and neck area. The medical records were retrospectively reviewed, identifying a total of 866 free flaps; this included seven double free flaps, all conducted by a single senior doctor at one medical institution.

Age, sex, smoking history, presence of comorbid diseases such as diabetes and hypertension, and history of preoperative radiation were investigated as risk factors that could potentially affect the survival of the free flap. The causes of reconstruction, pathology, primary sites, types of flaps, causes of postoperative vascular crisis, rate of emergency explorations, and treatment methods in case of failure of a free flap were analyzed. The success rates were compared according to the type of flap procedure performed, and complications arising from the donor site of the free flap were analyzed. For statistical analyses, IBM SPSS version 22 (IBM Corp., Armonk, NY, USA) was used. Chisquared tests were used to statistically determine the success rates for each flap; a value of $p<0.05$ was considered statistically significant.

\section{RESULTS}

The mean age of the cohort was 57.2 years (range, 6-91 years), including 661 men (76.9\%) and 198 women (23.1\%). Smoking history was identified in 451 patients (52.5\%), comorbid conditions in 369 patients (43.0\%), and preoperative radiotherapy

Table 1. Patient characteristics

\begin{tabular}{lc}
\hline Variable & Value \\
\hline Age $(\mathrm{yr})$ & $57.2(6-91)$ \\
$\geq 60$ & $396(46.1)$ \\
$<60$ & $463(53.9)$ \\
Sex & \\
Male & $661(76.9)$ \\
Female & $198(23.1)$ \\
Comorbid conditions & $369(43.0)$ \\
Diabetes & $133(15.4)$ \\
History of smoking & $451(52.5)$ \\
\hline Preoperative radiation therapy & $207(24.1)$ \\
\hline
\end{tabular}

Values are presented as mean (range) or number (\%). was received in 207 patients (24.1\%) (Table 1).

By comparing the failure rates of flaps according to risk factors that may affect their survival, the flap failure rates of patients over 60 years of age $(p=0.11)$, with comorbid diseases $(p=0.67)$, with diabetes $(p=0.30)$, with a history of smoking $(p=0.39)$, with preoperative radiation $(p=0.63)$, and of women $(p=0.26)$, were found to be higher in those who did not share these characteristics; however, there were no statistical differences (Table 2). The free flap method was performed on 797 patients (92.8\%) with malignant tumors and 62 patients $(7.2 \%)$ with non-malignant tumors. The pathological classifications of the malignant tumors were: squamous cell carcinoma in 703 (90.5\%), adenoid cystic carcinoma in 40 (5.1\%), mucoepidermoid carcinoma in 10 (1.3\%), verrucous carcinoma in four, malignant melanoma in three, adenocarcinoma in three, sarcomatoid carcinoma in three, papillary carcinoma in three, fibromyxoid sarcoma in two, and malignant fibrous histiocytoma in two cases. The causes of the non-malignant tumors included 28 postoperative complications, eight traumas, five benign tumors, three postirradiation complications, and three infections (Table 3). Regarding cancer staging, 502 cases were in stage 4,110 cases were in stage 3,140 cases were in stage 2, 37 cases were in stage 1 , and eight cases were in stage 0 .

The reconstructed sites included the oromandible $(n=355)$, oropharynx $(n=236)$, hypopharynx and larynx $(n=164)$, maxilla $(n=68)$, scalp $(n=9)$. Reconstructed sites in patients with malignant tumors included the oral cavity $(\mathrm{n}=317)$, oropharynx $(n=232)$, hypopharynx and larynx $(n=156)$, and maxilla $(n=59)$. The primary sites for malignant tumors were the tongue $(n=176)$, tonsils $(n=161)$, hypopharynx $(n=129)$. The free flaps used during reconstruction included the radial forearm free flap (RFFF, $n=557$ ), anterolateral thigh free flap (ALT$\mathrm{FF}, \mathrm{n}=200$ ), fibular osteocutaneous free flap (fibular OCFF,

Table 2. Variables regarding flap failure

\begin{tabular}{lcc}
\hline Variable & \% of flap failure & $p$-value \\
\hline Age $\geq 60$ yr & 1.5 & 0.11 \\
Age $<60$ yr & 3.1 & \\
Male & 2.1 & 0.26 \\
Female & 3.5 & \\
Comorbidity & 2.6 & 0.67 \\
No comorbidity & 2.2 & \\
Diabetes & 3.6 & 0.30 \\
No diabetes & 2.2 & \\
Smoking history & 3.0 & 0.39 \\
No smoking history & 1.9 & \\
Preoperative radiation therapy & 2.8 & 0.63 \\
No preoperative radiation therapy & 2.3 &
\end{tabular}


$\mathrm{n}=39$ ), latissimus dorsi musculocutaneous or muscle free flaps (LDMCFF or LDFF, $\mathrm{n}=24$ ), rectus abdominis musculocutaneous free flap (RAMCFF, $n=21$ ), jejunum free flap $(n=9)$, and dorsalis pedis free flap $(n=2)$. In addition, seven double free flap procedures were performed: fibular OCFF and RFFF

Table 3. Etiopathology of free flaps

\begin{tabular}{lc}
\hline Variable & Value (\%) \\
\hline Malignancy & $797(92.8)$ \\
Squamous cell carcinoma & $703(81.8)$ \\
Adenoid cystic carcinoma & $40(4.7)$ \\
Mucoepidermoid carcinoma & $10(1.2)$ \\
Verrucous carcinoma & $4(0.5)$ \\
Basaloid squamous cell carcinoma & $4(0.5)$ \\
Malignant melanoma & $3(0.4)$ \\
Adenocarcinoma & $3(0.4)$ \\
Sarcomatoid carcinoma & $3(0.4)$ \\
Papillary carcinoma & $3(0.4)$ \\
Fibromyxoid sarcoma & $2(0.2)$ \\
Malignant fibrous histiocytoma & $2(0.2)$ \\
Others & $20(2.4)$ \\
Non-malignancy & $62(7.2)$ \\
Postoperative complication & $28(3.3)$ \\
Trauma & $8(0.9)$ \\
Benign tumor & $5(0.6)$ \\
Post irradiation complication & $5(0.6)$ \\
Infection & $3(0.3)$ \\
Others & $13(1.5)$ \\
\hline
\end{tabular}

$(\mathrm{n}=2)$, fibular OCFF and LDMCFF $(\mathrm{n}=1)$, fibular OCFF and RAMCFF $(n=1)$, LDMCFF and deep circumflex iliac artery (DCIA) flap $(\mathrm{n}=1)$, LDMCFF and RAMCFF $(\mathrm{n}=1)$, and LDMCFF and LDMCFF $(\mathrm{n}=1)$ (Table 4).

The average size of radial forearm flap is $69.8 \mathrm{~cm}^{2}$ (range, $\left.7-176 \mathrm{~cm}^{2}\right)$, the average size of anterolateral thigh flap is 146.1 $\mathrm{cm}^{2}$ (range, 56-234 $\mathrm{cm}^{2}$ ), and the average size of fibular osteocutaneous flap is $68.1 \mathrm{~cm}^{2}$ (range, $21-171 \mathrm{~cm}^{2}$ ), The average length of bone was $9.2 \mathrm{~cm}$, and the average size of rectus abdominis flap was $111.0 \mathrm{~cm}^{2}$ (range, $63-220 \mathrm{~cm}^{2}$ ).

After surgery, vascular crisis occurred in 44 cases (5.1\%); emergency exploration was therefore performed. Regarding the causes, venous thrombosis was responsible in 23 cases (52.3\%); both vein and artery were thrombosed in six patients (13.6\%); there were five cases (11.4\%) of arterial thrombosis; and three cases (6.8\%) of carotid artery blow-out occurred due to infection at the anastomotic site. Among the 44 emergency explorations, 23 flaps were salvaged and 21 flaps could not be salvaged (salvage success rate: $52.3 \%$ ). As a result, the total success rate of the 866 free flap procedures performed over 30 years was $97.6 \%$ (845/866) (Table 5).

The failure rate according to the flap type was the highest in the ALTFF at $5.5 \%$ (11/200), followed by 5.1\% (2/39) in the fibular OCFF, $4.5 \%(1 / 21)$ in the RAMCFF, $4.4 \%(1 / 23)$ in the LDMCFF, and $1.1 \%$ in the RFFF (6/557). The RFFF showed a significantly lower failure rate than other types of flaps $(p<0.05)$, There were no significant differences in the failure rates between the other four types of flap (Table 6).

Table 4. Location and types of free flaps

\begin{tabular}{|c|c|c|c|c|c|c|c|}
\hline Free flap & Scalp & Maxilla & Oromandible & Oropharynx & Hypopharynx \& larynx & Others & Total \\
\hline Radial forearm & & 10 & 231 & 178 & 123 & 15 & 557 \\
\hline Anterolateral thigh & & 36 & 66 & 53 & 31 & 14 & 200 \\
\hline Fibular & & 2 & 33 & 1 & 1 & 2 & 39 \\
\hline Latissimus dorsi & 6 & 10 & 6 & & & 2 & 24 \\
\hline Rectus abdominis & & 8 & 10 & 3 & & & 21 \\
\hline Jejunal & & & & & 9 & & 9 \\
\hline DCIA osteocutaneous & & 2 & 2 & & & & 4 \\
\hline Scapular fascial & & 1 & 2 & & & & 3 \\
\hline Lateral arm & & & 1 & 1 & & & 2 \\
\hline Dorsalis pedis & & & 2 & & & & 2 \\
\hline Serratus anterior & 1 & & & & & & 1 \\
\hline Omental & 1 & & & & & & 1 \\
\hline Temporoparietal & 1 & & & & & & 1 \\
\hline Groin & & & 1 & & & & 1 \\
\hline Helical & & & & & & 1 & 1 \\
\hline Total & 9 & 68 & 355 & 236 & 164 & 34 & 866 \\
\hline
\end{tabular}

DCIA, deep circumflex iliac artery. 
Table 5. Causes of vascular crisis

\begin{tabular}{lc}
\hline Variable & Value $(n=44)$ \\
\hline Vascular crisis & $23(52.3)$ \\
Vein thrombosis & $6(13.6)$ \\
Vein \& arterial thrombosis & $3(6.8)$ \\
Arterial thrombosis & $6(13.6)$ \\
Anastomosis site blow out due to infection & $6(13.6)$ \\
Others & \\
Success of re-anastomosis & 16 \\
Vein thrombosis & 2 \\
Arterial thrombosis & 5 \\
Carotid blow out due to infection & 1 \\
Sting injury of needle aspiration & $23(52.3)$ \\
Salvage success rate &
\end{tabular}

Values are presented as number (\%) or number.

Of the 21 cases of failed emergency exploration, 13 patients $(61.9 \%)$ received new free flaps, seven $(33.3 \%)$ with a poor general condition underwent reconstruction using pedicled myocutaneous flaps, and one patient with a small defect in the tonsil area was treated via direct closure.

Complications at the donor site occurred in $13.3 \%$ of cases and included wound disruption, partial loss of skin graft, seroma formation, and tendon exposure; $35.3 \%$ of complications occurred in LDFFs, $15.1 \%$ in RFFFs, and 5.5\% in ALTFFs. Partial loss of skin graft $(83.3 \%)$ and seroma (16.7\%) occurred at the donor site of LDFFs. Surgical treatment was required for $73.9 \%$ of the complications; the rest were improved with conservative treatment.

\section{DISCUSSION}

Following the introduction of free flap surgery in the 1970s, the development of techniques and instruments for microsurgery, as well as the understanding of vascular anatomy, began to increase. As a result, various new flaps were developed and development of microsurgical reconstruction has allowed plastic surgeons to reconstruct even the most complex defects in head and neck areas. In addition, it enables the oncologic surgeons to perform wide resection which can reduce the recurrence rate.

Microvascular free tissue transfer takes a long time to operate, and donor site morbidity cannot be avoided; thus, if the operation fails, great distress is caused to both the surgeon and patient. From the stage of operation planning, in order to increase the success rate, factors which could affect the survival of the flap should be considered [7-11]. As confirmed by the results of this study, Simpson et al. [11] argued that factors other than the surgeon's surgical experience did not significantly affect the
Table 6. Flap failure rate of flap types

\begin{tabular}{lc}
\hline Free flap & Flap failure rate (No./No.) \\
\hline Radial forearm & $1.1(6 / 557)$ \\
Anterolateral thigh & $5.5(11 / 200)$ \\
Fibula & $5.1(2 / 39)$ \\
Rectus abdominis & $4.5(1 / 21)$ \\
Latissimus dorsi & $4.4(1 / 23)$ \\
Total & $2.4(21 / 866)$ \\
\hline
\end{tabular}

success rate of flaps. Similarly, Kim et al. [9] reported that prior radiotherapy or neck dissection did not affect the success rate of the flap, even when the free flap was performed for recurrent head and neck cancer.

Monitoring of the flap condition after surgery was confirmed by visual examination and palpation in cases that could be visually confirmed; at sites such as the hypopharynx and larynx that could not be seen with direct vision, examination was performed using an endoscope. Wu et al. [3] stated that flap monitoring was performed at 1-hour intervals on postoperative day (POD) 1, 2-hour intervals on POD 2, and 4-hour intervals on PODs 3-7. In our study, however, flap monitoring was performed at 6-hour intervals until POD 3, 8-hour intervals on PODs 4-7, and twice a day on PODs 8-14. Devine et al. [10] reported that salvage was successful in $88.6 \%$ of cases if emergency exploration was performed within 24 hours after the flap compromise was discovered. At the beginning of microsurgical reconstruction, exploration was performed only in cases showing definitive blood circulation abnormality of the flap. However, this approach resulted in high rates of salvage failure and this experience stressed the importance of early exploration. Therefore, from the early the 1990s onward, exploration was performed as soon as possible if any abnormality in the blood circulation of the flap was suspected. Antithrombotic agents are not usually used after surgery. However, prostagrandin-E1 (alprostadil) was used when emergency exploration was performed due to vascular crisis of the flap.

In previous studies, the probability of performing emergency exploration due to vascular crisis was reported to be $6.2 \%$ to $20 \%$ and venous insufficiency was the most common cause [2$6,10-14]$. In this study, vascular crisis occurred in $5.1 \%$ of cases; likewise, venous insufficiency $(52.3 \%)$ was the most common cause.

Of the 21 cases of complete flap loss, 13 reconstructions using a free flap were performed; all were successful. Seven cases were reconstructions using pedicled flaps due to the poor general condition of the patient; the remaining case with small tissue defects in the tonsil was treated with direct closure.

The strengths and weaknesses of RFFF (557 cases, 64.3\%) and 
ALTFF (200 cases, 23.1\%), which were used to reconstruct soft tissue defects in the head and neck, were compared. Anatomically, RFFF has a larger arterial diameter than ALTFF and it contains two venae comitantes and cephalic veins. These characteristics of RFFF give advantage over ALTFF during anastomosis. Even at the flap elevation stage, most ALTFF perforators from the lateral circumflex femoral artery are musculocutaneous perforators rather than septocutaneous perforators. This vascular anatomy makes dissection more difficult than for RFFF. Additionally, while dissecting the perforator, exceptional care should be taken especially at the location where the perforator enters the cutaneous flap. At this location, the diameter of the perforator is small and can be easily damaged by traction kinking Nevertheless, ALTFF has the advantage of harvesting a greater amount of tissue than RFFF, as well as the direct primary closure is possible if the width of donor site is less than $8 \mathrm{~cm}$. Several studies favoring the benefits of ALTFF reported no significant difference in flap survival rates between RFFF and ALTFF [15-18]; however, in this study, the success rate of RFFF (98.9\%) was significantly higher than of ALTFF (94.5\%) $(p<0.01)$.

Mandibular reconstruction was performed using fibular OCFF (33 cases) or DCIA osteocutaneous flaps ( 2 cases); two fibular OCFF failed. The failure rate of fibular OCFF performed on mandibular area reconstructions was $6.1 \%$, higher than at other sites. Higher failure rate of mandibular reconstruction using fibular OCFF was also reported in other studies $[6,19,20]$. Two unique characteristics are considered to be the cause of higher failure rate. One is difficult flap insetting procedure due to the complex structure of the mandible. The other is technical difficulty of anastomosis due to the size discrepancy between the diameter of the lower extremity vessels and cervical vessels.

Double free flap, using two free flaps simultaneously, has innately higher possibility of failure because of its longer operation time and technical complexity. However, a double free flap needs to be performed for composite tissue defects in the head and neck, or when a large volume is required due to a wide range of tissue defects [21]. The authors performed double free flaps on seven patients; five underwent simultaneous reconstruction of the mandibular defect and structure in the oral cavity, while the other two had extensive soft tissue defects. Wu et al. [3] reported that double free flaps were performed in 101 cases. While the re-exploration rate was significantly higher than of single free flaps, there was no statistical difference in flap failure rate. In this study, two cases of venous thrombosis occurred out of the seven double free flap procedures performed, and one of them could not be salvaged with an exploration.
Table 7. Total flap success rates in recent literatures

\begin{tabular}{llcc}
\hline Author (year) & Country & $\begin{array}{c}\text { Flap success } \\
\text { rate (\%) }\end{array}$ & $\begin{array}{c}\text { No. of data } \\
\text { enrolled }\end{array}$ \\
\hline Wei et al. (2001) [5] & Taiwan & 96.6 & 1,235 \\
Eckardt et al. (2003) [4] & Greece & 94.0 & 500 \\
Nakatsuka et al. (2003) [6] & Japan & 95.8 & 2,372 \\
Bianchi et al. (2009) [2] & Italy & 96.0 & 352 \\
Wu et al. (2014) [3] & Taiwan & 96.2 & 1,918 \\
Current study (2020) & Korea & 97.6 & 866 \\
\hline
\end{tabular}

The donor site complications occurred in $15.1 \%$ of RFFF. Majority of them were related to the loss of the skin graft and were treated with a local flap or additional skin graft. Since RFFF requires sacrifice of the radial artery, it is important to perform the Allen test to assess for any abnormalities in the ulnar artery before surgery [22]. In this study, one case of arterial insufficiency occurred in the hand after surgery, resolved by performing a vein graft on the radial artery. The number of complications at the donor site was lowest in ALTFF, followed by RFFF, which was similar to that of the fibula. Although RFFF can cause functional and aesthetic disturbance in patients with hypertrophic scarring, pigmentation, sensory disturbance, and social stigma, the donor site does not affect normal daily activity [23].

In prior literature with suitable cohort sizes (over 300 cases) published after the 2000s, the success rates of free flaps performed for head and neck reconstructions was reported to be between $94 \%$ and $96.6 \%$ [2-6]. The authors analyzed 866 free flaps, reporting a success rate of $97.6 \%$; in patients with malignant tumors, the success rate was $97.8 \%$ (Table 7).

In conclusion, if the patient's general condition is stable, the free flap is a reliable method for head and neck reconstruction. In order to increase the success rate of the free flap, complete surgical equipment, manpower, and experienced microsurgeons are required; if any abnormality in the blood circulation of the flap is found after surgery, exploration should be performed as soon as possible.

\section{NOTES}

\section{Conflict of interest}

No potential conflict of interest relevant to this article was reported.

\section{Ethical approval}

The study was approved by the Institutional Review Board of Kangdong Sacred Heart Hospital (IRB No. 2020-09-003) and performed in accordance with the principles of the Declaration 
of Helsinki. Informed consent was waived because of the retrospective nature of the study and the analysis used anonymous clinical data.

\section{ORCID}

Joong Min Suh https://orcid.org/0000-0002-3475-0091 Chul Hoon Chung https://orcid.org/0000-0003-3722-1838 Yong Joon Chang https://orcid.org/0000-0003-4966-6543

\section{Author contribution}

Conceptualization: CHC, YJC. Data curation: JMS, CHC, YJC. Formal analysis, software: JMS. Methodology: JMS, CHC, YJC. Project administration: YJC. Investigation: JMS, CHC. Visualization: JMS. Supervision, validation: CHC. Writing - original draft: JMS. Writing - review \& editing: JMS, CHC. Approval of final manuscript: all authors.

\section{REFERENCES}

1. Daniel RK, Taylor GI. Distant transfer of an island flap by microvascular anastomoses: a clinical technique. Plast Reconstr Surg 1973;52:111-7.

2. Bianchi B, Copelli C, Ferrari S, Ferri A, Sesenna E. Free flaps: outcomes and complications in head and neck reconstructions. J Craniomaxillofac Surg 2009;37:438-42.

3. Wu CC, Lin PY, Chew KY, Kuo YR. Free tissue transfers in head and neck reconstruction: complications, outcomes and strategies for management of flap failure: analysis of 2019 flaps in single institute. Microsurgery 2014;34:339-44.

4. Eckardt A, Fokas K. Microsurgical reconstruction in the head and neck region: an 18-year experience with 500 consecutive cases. J Craniomaxillofac Surg 2003;31:197-201.

5. Wei FC, Demirkan F, Chen HC, Chuang DC, Chen SH, Lin $\mathrm{CH}$, et al. The outcome of failed free flaps in head and neck and extremity reconstruction: what is next in the reconstructive ladder? Plast Reconstr Surg 2001;108:1154-60.

6. Nakatsuka T, Harii K, Asato H, Takushima A, Ebihara S, Kimata Y, et al. Analytic review of 2372 free flap transfers for head and neck reconstruction following cancer resection. J Reconstr Microsurg 2003;19:363-8.

7. Classen DA, Ward H. Complications in a consecutive series of 250 free flap operations. Ann Plast Surg 2006;56:557-61.

8. Suh JD, Sercarz JA, Abemayor E, Calcaterra TC, Rawnsley JD, Alam D, et al. Analysis of outcome and complications in 400 cases of microvascular head and neck reconstruction. Arch Otolaryngol Head Neck Surg 2004;130:962-6.

9. Kim HS, Chung $\mathrm{CH}$, Chang YJ. Free-flap reconstruction in recurrent head and neck cancer: a retrospective review of 124 cases. Arch Craniofac Surg 2020;21:27-34.

10. Devine JC, Potter LA, Magennis P, Brown JS, Vaughan ED. Flap monitoring after head and neck reconstruction: evaluating an observation protocol. J Wound Care 2001;10:525-9.

11. Simpson KH, Murphy PG, Hopkins PM, Batchelor AG. Prediction of outcomes in 150 patients having microvascular free tissue transfers to the head and neck. Br J Plast Surg 1996;49: 267-73.

12. Jones NF, Johnson JT, Shestak KC, Myers EN, Swartz WM. Microsurgical reconstruction of the head and neck: interdisciplinary collaboration between head and neck surgeons and plastic surgeons in 305 cases. Ann Plast Surg 1996;36:37-43.

13. Pohlenz P, Klatt J, Schon G, Blessmann M, Li L, Schmelzle R. Microvascular free flaps in head and neck surgery: complications and outcome of 1000 flaps. Int J Oral Maxillofac Surg 2012;41:739-43.

14. Kubo T, Yano K, Hosokawa K. Management of flaps with compromised venous outflow in head and neck microsurgical reconstruction. Microsurgery 2002;22:391-5.

15. Camaioni A, Loreti A, Damiani V, Bellioni M, Passali FM, Viti C. Anterolateral thigh cutaneous flap vs. radial forearm freeflap in oral and oropharyngeal reconstruction: an analysis of 48 flaps. Acta Otorhinolaryngol Ital 2008;28:7-12.

16. Oranges CM, Ling B, Tremp M, Wettstein R, Kalbermatten DF, Schaefer DJ. Comparison of anterolateral thigh and radial forearm free flaps in head and neck reconstruction. In Vivo 2018; 32:893-7.

17. Young AMH, Bache S, Segaren N, Murphy S, Maraka J, Durrani AJ. Free flap selection and outcomes of soft tissue reconstruction following resection of intra-oral malignancy. Front Surg 2019;6:53.

18. Liu WW, Li H, Guo ZM, Zhang Q, Yang AK, Liu XK, et al. Reconstruction of soft-tissue defects of the head and neck: radial forearm flap or anterolateral thigh flap? Eur Arch Otorhinolaryngol 2011;268:1809-12.

19. Shanker MK, Rajan A, Hemant B, Kumar DA. Outcome of 1000 free flap head and neck reconstructions at a tertiary cancer care institute in India. Eur J Plast Surg 2021;44:25-32.

20. Kroll SS, Schusterman MA, Reece GP, Miller MJ, Evans GR, Robb GL, et al. Choice of flap and incidence of free flap success. Plast Reconstr Surg 1996;98:459-63.

21. Guillemaud JP, Seikaly H, Cote DW, Barber BR, Rieger JM, Wolfaardt J, et al. Double free-flap reconstruction: indications, challenges, and prospective functional outcomes. Arch Otolaryngol Head Neck Surg 2009;135:406-10.

22. Allen EV. Thromboangiitis obliterans: methods of diagnosis of chronic arterial lesions distal to the wrist with illustrative cases. Am J Med Sci 1929;178:237-44. 
23. Huang $\mathrm{CH}$, Chen HC, Huang YL, Mardini S, Feng GM. Comparison of the radial forearm flap and the thinned anterolateral thigh cutaneous flap for reconstruction of tongue defects: an evaluation of donor-site morbidity. Plast Reconstr Surg 2004; 114:1704-10. 